\title{
Why so little progress in therapeutic thrombolysis? The current state of the art and prospects for improvement
}

\author{
Victor Gurewich ${ }^{1}$
}

Published online: 18 April 2015

(c) The Author(s) 2015. This article is published with open access at Springerlink.com

\section{Introduction}

Thrombosis is a major contributor to disability and mortality worldwide and is estimated to be the cause of one in four deaths [1]. It is the pathology responsible for triggering acute myocardial infarction (AMI) and ischemic stroke, and is the primary cause of venous thromboembolism. The pathogenesis has been well-studied starting with Virchow more than 150 years ago [2], and its prevention by anti-thrombotic drugs has made much progress, culminating most recently in the approval of new oral anticoagulants which are specific inhibitors of thrombin or factor Xa.

By contrast, treatment by thrombolysis, the only pharmacological means available, has remained at a near standstill since Genentech first applied for FDA approval of tissue plasminogen activator (tPA) in 1983. A replacement for streptokinase (SK) had been long-sought, but after early successes in AMI, tPA use has since been on the decline.

Instead, percutaneous coronary intervention (PCI) has become the treatment of choice in AMI, despite its technical, time-consuming, and costly demands. Pre-treatment with tPA to hasten coronary reperfusion, so-called facilitated PCI, was found to increase PCI complications including intracranial hemorrhage (ICH) making the two incompatible [3]. In ischemic stroke, tPA has been estimated to be used in only 2-5\% of patients over-all [4], and in pulmonary embolism, bleeding and stroke complications

Victor Gurewich

vgurewich@tsillc.net

1 Vascular Research Laboratory, Mt Auburn Hospital, Harvard Medical School, Cambridge, MA, USA limited its use to the minority of patients with hemodynamic decompensation [5].

The recent trend in stroke has also begun to favor the use of endovascular devices with three major trials in ischemic stroke published in the first 2 months of 2015 in the NEJM [6-8]. In this same period, a clinical trial of catheter-directed ultrasound was also published which showed a significant acceleration of the thrombolysis rate by this method [9].

These device reperfusion techniques require bringing the patient to a qualified facility which inevitably consumes precious time for conditions in which "minutes are myocardium or neurons." An optimal treatment for these time-sensitive conditions can only be one that can be brought to the patient and is easily and safely administered. The resort to alternative methods is a reflection on the inadequacy of current thrombolysis, a problem that was foretold by the results with tPA in major clinical trials.

\section{A brief review of thrombolysis with tPA}

TPA was the first fibrin-specific thrombolytic [10] and replaced SK, which was non-specific, causing extensive hematological side effects, and which was an indirect plasminogen activator. By contrast, tPA is a direct activator and one that is strongly promoted by fibrin to which it has a high affinity [11]. In the same year, this property was shown to be sufficient to purify tPA directly from human plasma in a single step [12].

In 1983, tPA was successfully produced by recombinant technology by Genentech [13], and AMI was selected as the first disease target. It was anticipated that because of its fibrin-specific mode of action, tPA would prove far more effective than SK, as also suggested by preliminary trials 
[14]. However, their difference turned out to be so marginal that it required an unprecedented total of 94,720 patients to arrive at a statistically significant $p$ value in comparative clinical trials.

\section{Summary of the three mega trials of tPA versus SK in AMI}

1. GISSI-2 [15]

In a multicenter trial, 12,490 patients were randomized to either single-chain tPA (100 mg infused iv over $3 \mathrm{~h}$ ) or a standard dose of SK. No difference in the 30 days death rate was found, which was $9 \%$ for tPA and $8.6 \%$ for SK. The incidence of congestive heart failure, the second endpoint, was also not different. The complication rate of stroke (1.1 and $0.9 \%$ for tPA and SK respectively) and major bleeding (0.5 and $1 \%)$ were also similar.

2. ISIS-3 [16]

A total of 41,229 patients were randomized to standard doses of double chain tPA (Duteplase), SK, or anisoylated SK (APSAC). All patients received aspirin and half of each group received sc heparin starting at $4 \mathrm{~h}$ post thrombolysis. The outcome of the study was similar to that of the previous one with a 35 days mortality of $10.3 \%$ for tPA, $10.6 \%$ for SK, and $10.5 \%$ for APSAC. A significant excess of total stroke with tPA was, however, found, being $1.39 \%$ for tPA and $1.04 \%$ for SK $(\mathrm{p}<0.01)$.

Once again, no explanation for the surprising lack of clinical benefit from tPA over SK was offered.

3. The GUSTO study [17]

The final multinational study was with 42,021 patients divided into four groups, two with SK plus either sc or iv heparin and two with TPA and iv heparin, in one of which the TPA was administered by a new accelerated regimen. Only in the group given tPA by the accelerated regimen, was there a statistically significant difference found. The 30 day mortality in this group was $6.3 \%$ with tPA and $7.2 \%$ for SK $(\mathrm{p}<0.001)$. The ICH incidence again was significantly $(\mathrm{p}=0.03)$ higher with tPA than SK, as was the incidence of "moderate or worse" bleeding $(\mathrm{p}=0.02)$.

The finding of a significant difference in the result from this trial was met with some skepticism. In a commentary by three prominent experts, it was concluded that there was "at most, a small absolute difference... in both lives saved and major complications between tPA and SK" [18]. The GUSTO trial results also underwent a Bayesian analysis which found them to be inconclusive and that "the clinical superiority of tPA over SK remains uncertain" [19].
Nevertheless, tPA was given approval for AMI treatment after the GUSTO trial and tPA or one of its longer half-life derivatives, has remained the thrombolytic of choice ever since becoming essentially synonymous with thrombolysis. In 1996, tPA was also approved for the treatment of ischemic stroke.

The unprecedented number of patients that were required to gain approval for a new thrombolytic had its adverse consequences. The experience not only discouraged further investment in thrombolysis by industry, but it also dampened scientific interest. It was believed that the trials showed that all activators must be more or less comparable and that the difference between a fibrinspecific and a non-specific activator was less important than had been believed. It was as if the limits of what was possible with thrombolysis had been reached.

In retrospect, a crucial alternative interpretation was missed. This was that the very nature of tPA's fibrinspecific mode of action limited its fibrinolytic effect and conversely, that it was the non-specific mode of action of SK that gave it an unrestricted plasminogen activating effect which gave it a comparable efficacy. The activators' net effect was similar but for very different reasons. The more limited fibrinolytic effect of tPA had been previously implied by the finding that its fibrin-dependent plasminogen activation was different and complementary to that of the other natural plasminogen activator prourokinase (proUK) [20].

\section{The biological pathway of fibrinolysis and its lessons}

Since all therapeutic thrombolysis utilizes the endogenous plasminogen-plasmin pathway, endogenous fibrinolysis provides a useful guide to its function and utilization. For hemostasis, fibrin must be sufficiently stiff to serve as a meshwork to prevent bleeding but at the same time it must be capable of being lysed when vital blood flow is threatened by the same meshwork. Plasmin is responsible for fibrinolysis and does so by cleaving the polymer into two major soluble fibrin degradation products [21], a remarkably efficient solution to the problem. Plasminogen, the proenzyme precursor of plasmin, is available in relatively high abundance in plasma $(\sim 2 \mu \mathrm{M})$, more than sufficient for its therapeutic utilization.

Plasmin is relatively non-specific and hydrolyzes other substrates including three clotting factors, and can induce a hemorrhagic diathesis. To make it more fibrin-specific, two additional factors have evolved. First plasmin(ogen) targets fibrin by binding directly to certain lysine binding sites [22]. This both targets and promotes its fibrinolytic activity. Unbound plasmin, by contrast, induces little 
fibrinolysis, as evidenced by microplasmin which has no fibrin binding domains and induces little fibrin degradation but retains a full fibrinogenolytic effect [23]. Second, the two endogenous activators of plasminogen, tPA and proUK, have high specificities for fibrin-bound plasminogen over that which is not bound. As a result, the biological system is highly fibrin specific and proteolysis is additionally limited to the fibrin clot environment by certain inhibitors in the ambient plasma. Importantly, the endogenous system utilizes both activators for fibrinolysis rather than just one as has been the custom in therapeutic thrombolysis.

\section{The sequential and complementary mechanisms of fibrin-dependent plasminogen activation by tPA and proUK}

When intravascular thrombosis occurs, tPA stored in the vessel wall at that site is released, binds to the thrombus, and initiates fibrin degradation. This is mediated by tPA's high fibrin affinity for a specific site on the D-domain of intact fibrin $[11,24]$ that is adjacent to fibrin-bound plasminogen. This ternary complex promotes plasminogen activation by tPA more than 1000-fold [25] and efficiently initiating degradation of the fibrin surface. This creates new plasminogen binding sites [26], and plasminogen bound to these new sites is activated preferentially by proUK and its activated form urokinase (UK). Specifically, proUK has a high substrate affinity for plasminogen bound to a triple carboxyterminal lysine high affinity site on the fibrin E-domain of degraded fibrin [27]. Plasminogen at this site undergoes a conformational change which promotes proUK's intrinsic activity more than 250 -fold, giving it an activity equivalent to that of UK [28]. A similar promotion of proUK's intrinsic activity by plasminogen bound to solid-phase fibrin after its degradation was also shown in another study [29].

This activation of plasminogen by proUK is accompanied by reciprocal activation of proUK by plasmin [30] that is associated with a hypercatalytic transitional state between proUK and UK [31]. Thereafter, UK activates the remaining plasminogen on degraded fibrin. The activation of proUK during lysis adds to its fibrinolytic effect as illustrated by a study with an inactivatable, plasmin-resistant mutant proUK which was 100 -fold less effective in clot lysis [32]. ProUK/UK, therefore, has a dual plasminogenactivating function in fibrinolysis. By contrast, tPA undergoes no functional change since its one and two-chain forms have similar fibrinolytic properties [33]. This relatively more limited effect of tPA was observed in an earlier study in which the rates of fibrin-specific clot lysis ( $<10 \%$ fibrinogenolysis) in a plasma milieu by tPA and proUK were compared. The lysis rate by proUK was consistently twice that of tPA [34], a finding which indicated that proUK activated twice as many fibrin-bound plasminogens.

These findings suggest that there are a total of three different plasminogen binding sites available on fibrin, one on intact fibrin activated by tPA and two additional ones on degraded fibrin activated by proUK and UK, consistent with the 2:1 ratio of their lysis rates. This same number of plasminogens was documented in a previous study in which the molar amounts of plasminogen on intact and degraded fibrin were measured. There were $23.4 \mathrm{nmol} / \mathrm{l}$ of plasminogen found on intact fibrin and $61.9 \mathrm{nmol} / \mathrm{l}$ on degraded fibrin for a total of three [35].

The fibrinolytic activities of tPA and proUK are, therefore, promoted by different fibrin domains which are the $\mathrm{D}$ and $\mathrm{E}$ domains respectively. This was also shown in a kinetic study with soluble fibrin fragments D and E in which plasminogen activation was measured. Plasminogen activation by tPA was promoted only by fragment $\mathrm{D}$, whereas that by proUK was promoted only by fibrin fragment E [36], making the activators complementary in their fibrin-dependent plasminogen activating effects.

\section{Clinical implications}

These fibrinolytic differences also affect the etiology of the hemorrhagic side effects of tPA and proUK. Hemostatic fibrin, being protected from fibrinolytic degradation, remains intact and consequently is much more vulnerable to lysis by tPA than by proUK. This is not a problem physiologically because tPA normally circulates as an inactive complex with its inhibitor, plasminogen activator inhibitor-1 (PAI-1), but PAI-1 is at far too low a concentration for therapeutic amounts of tPA, leaving hemostatic fibrin unprotected. This explains the unexpected finding of a significantly higher incidence of ICH by tPA than by nonspecific SK in the mega trials [16, 17]. The lysis of hemostatic fibrin by tPA was previously cited as the principle cause $[37,38]$. Therefore, bleeding is a direct consequence of tPA's mechanism of action of targeting intact fibrin to initiate lysis, explaining also why its therapeutic doses need to be limited.

This tPA effect was studied experimentally in a dog model in which blood loss from standardized hemostatically sealed fresh injury sites was measured. Blood loss by tPA was tenfold higher than that by proUK (mean $40 \mathrm{vs}$ $4 \mathrm{ml}, \mathrm{p}=0.026$ ) at equivalent fibrinolytic doses [39], a finding consistent with the differences in their mechanisms of fibrin-dependent plasminogen activation.

The etiology of proUK's bleeding side effects is quite different. ProUK is vulnerable to conversion to UK at therapeutic concentrations in plasma due to plasmin generation at these concentrations. UK compounds this 
problem by generating much more plasmin inducing a hemorrhagic diathesis with degradation of clotting factors like fibrinogen, as seen in the proUK clinical trials [40]. It was for this reason that, proUK was denied marketing approval, in Europe. To address this problem, a mutant form of proUK was developed (see below).

\section{Synergistic fibrinolysis by tPA and proUK in vitro and in vivo}

When IPA and proUK were used together versus either activator alone, a synergistic effect was observed in clot lysis experiments in a plasma milieu [41] which was attributed to their complementary modes of action [20]. Nevertheless, the synergistic effect of tPA and proUK has continued to remain controversial since it was contested by other investigators [42].

The effect of a sequential combination was also evaluated clinically in a multicenter study of patients with AMI. In the PATENT trial, 101 patients were treated with a small bolus of tPA followed by a modest infusion of proUK [43]. The first 10 patients were given a $10 \mathrm{mg}$ bolus which turned out to be excessive. The remainder were given a $5 \mathrm{mg}$ bolus of tPA ( $5 \%$ of the standard dose) followed by a proUK infusion of $40 \mathrm{mg} / \mathrm{h}$ (50\% of the standard rate) for $90 \mathrm{~min}$. A TIMI 2-3 coronary patency rate of $77 \%$ and a TIMI 3 patency rate of $60 \%$ were obtained, with no reocclusions or strokes and a mortality of $1 \%$. The patency rate obtained was comparable to that reported for the best of the four groups in the GUSTO trial [44], in which the mortality was $6.3 \%$ with $1.5 \%$ strokes [17].

Despite the success of the PATENT trial, no follow-up study was possible since Farmitalia, the sponsor, was sold soon after and proUK development ceased, and was discontinued by Gruenenthal a little later after their submission for marketing approval of proUK was denied due to the UK bleeding complications.

\section{The clinical experience with proUK in brief}

ProUK, the native, precursor of UK, was first isolated from human urine and identified to be a single-chain proenzyme in 1981 [12, 45]. It was shown to have a fibrin-specific mode of action, in contrast to UK, despite having no fibrin clot affinity [46]. After several small clinical studies with proUK for different indications, phase III clinical trials in AMI were done with recombinant proUK from $E$. coli by Gruenenthal $[47,48]$. An efficacy comparable to that of other activators was found but with a lower rate $(0-5 \%)$ of rethrombosis and no hematological evidence of thrombin generation [49], in contrast to tPA. However, at the therapeutic concentrations of proUK used, its relatively high intrinsic activity [50] induced systemic plasmin generation which converted proUK to UK, inducing extensive (80-90\%) fibrinogenolysis [40]. As noted above, due to this problem, marketing authorization for proUK was denied by the European Medicines Agency in 1998, "on the basis of the higher incidence of hemorrhagic strokes," and proUK development was terminated.

Since proUK had unique properties and therapeutic potential, especially in combination with tPA, it was decided to address the problem of its vulnerability to UK conversion by recombinant technology.

\section{Mutant proUK (HisproUK)}

Structure-function studies revealed a flexible loop in the catalytic domain of proUK with a charged residue (Lys 300 ) at its tip which was shown to be responsible for proUK's relatively high intrinsic activity [51]. By reducing the charge at this position, the intrinsic activity could be modulated [52]. A single site mutant (Lys300 $\rightarrow$ His) was eventually selected which had a fivefold lower intrinsic activity but a preserved two-chain enzymatic activity and other properties of proUK were also preserved. The HisproUK mutant was stable in plasma at a fivefold higher concentration than native proUK.

In a dog model, HisproUK induced effective lysis of venous thromboemboli which was associated with surprisingly little bleeding from hemostatic sites [53]. This phenomenon was tested in a second dog study against an arterial thrombus. HisproUK induced a vessel patency rate equivalent to that of tPA, but with tenfold less blood loss from fresh hemostatic sites than tPA [39], as also described above. The low bleeding rate was related to a novel inhibitor complex between HisUK and plasma $\mathrm{C} 1$ esterase inhibitor (C1INH) in the plasma of these dogs, which inhibited HisUK mediated side effects.

\section{The C1INH effect}

The same HisUK:C1INH inhibitor complex was found in human plasma after incubation with HisUK. In clot lysis studies in a plasma milieu, C1INH inhibited fibrinogenolysis by HisproUK without interfering with fibrinolysis [54]. Since C1INH is available as a pharmacological agent, clot lysis experiments were done with supplemental C1INH sufficient to double and triple its physiological concentration. At these higher concentrations, a maximum rate of clot lysis by HisproUK could be induced without causing the fibrinogen degradation, which otherwise accompanied such doses [55]. The C1INH prevention of fibrinogenolysis, but 


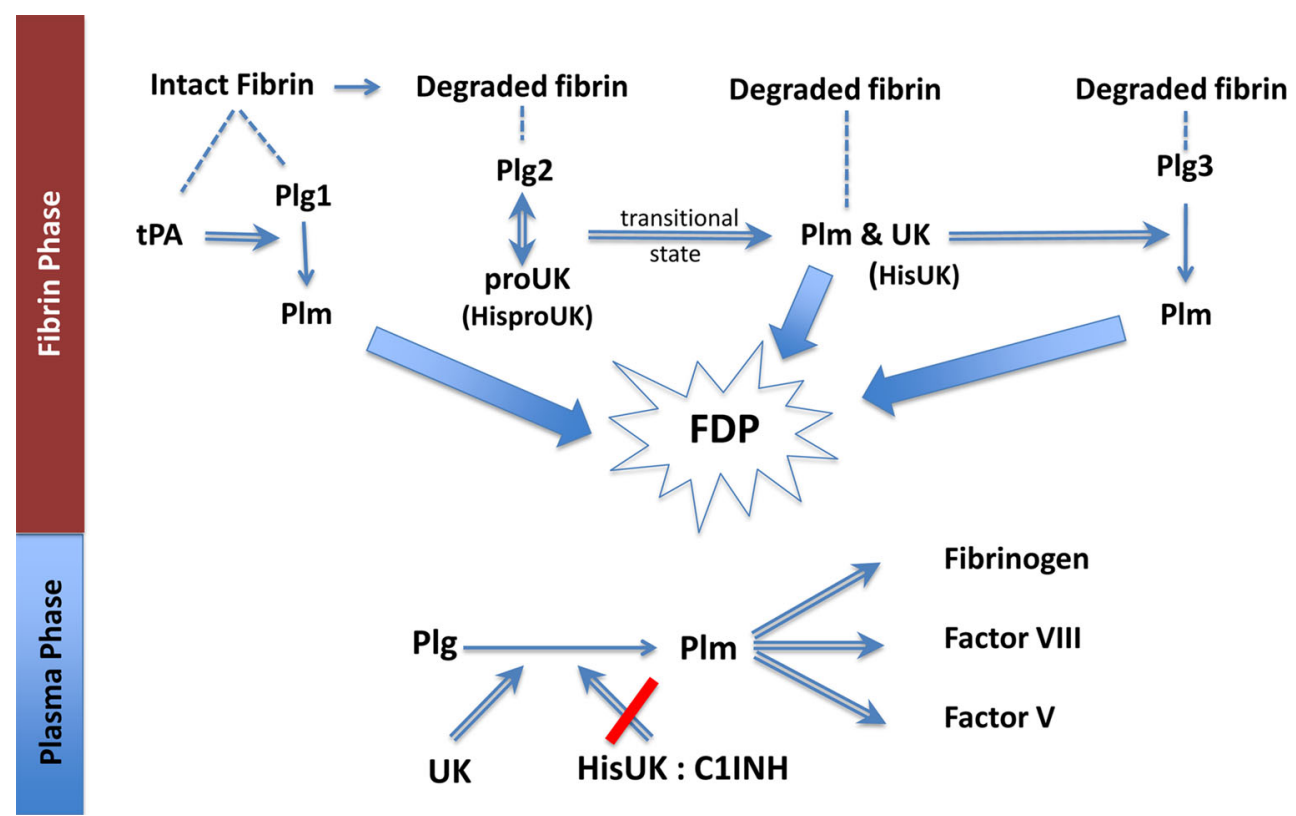

Fig. 1 Fibrinolysis by the sequential modes of action of tPA and proUK or HisproUK: tPA binds (dashed line) to intact fibrin adjacent to plasminogen $(\mathrm{Plg} 1)$, forming a ternary complex and activating $\mathrm{Plg} 1$ to plasmin (Plm). This initiates fibrin degradation resulting in the creation of new plasminogen binding sites on fibrin. Plg2 binds to a high-affinity binding site on the fibrin E (FFE) domain, which induces a special conformational shape change in $\mathrm{Plg} 2$ that enables it to be activated by the intrinsic activity of HisproUK. This HisproUK:plasminogen complex results in Plm formation and the reciprocal activation (double arrow) of HisproUK $\rightarrow$ HisUK, associated with

not fibrinolysis, by HisproUK is a unique property for a plasminogen activator.

$\mathrm{C} 1 \mathrm{INH}$ is a relatively abundant $(\sim 250 \mu \mathrm{g} / \mathrm{ml})$ plasma inhibitor, whereas plasminogen activator inhibitor-1 (PAI1), the principal inhibitor of $\mathrm{UK}$, is present only at a nanogram concentration $(21 \pm 7 \mathrm{ng} / \mathrm{ml})$. Since HisproUK is activated to HisUK during clot lysis, it's inhibition by plasma C1INH helps prevent the bleeding complications that occurred due to UK (see Fig. 1). Since C1INH is also an acute phase reactant, it is apt to be present at higher concentrations in patients with AMI, stroke or thromboembolism, so that supplemental C1INH is less likely to be needed, especially at the lower HisproUK doses needed with the synergistic combination.

\section{Testing fibrinolytic synergy of tPA and HisproUK}

In a clot lysis model in a plasma milieu, the dose of tPA or HisproUK alone which induced the shortest clot lysis time possible was established. Thereafter, the fractional dose combination that gave a comparable lysis time was selected and consisted of 3-6\% of the tPA dose combined with $40 \%$ that of the HisproUK. These fractional doses were almost identical to those of tPA and proUK in the clinical a transitional state that is hypercatalytic. UK, being a non-specific enzyme, then activates the remaining fibrin-bound plasminogen (Plg3). The three Plm together complete fibrin degradation (thick arrows) forming soluble fibrin degradation products (FDP). In the Plasma Phase, when UK diffuses off the fibrin clot, if it is not inhibited, it will activate plasma $\mathrm{Plg}$ to $\mathrm{Plm}$, resulting in the degradation of 3 clotting factors, among other side effects. When HisproUK is used in place of proUK, this non-specific effect is prevented due to HisUK being inhibited by $\mathrm{C} 1 \mathrm{INH}$, whose plasma concentration is $>1000$-fold greater than that of the UK inhibitor

PATENT trial, which were 5 and $40 \%$, as described above. This similarity between the findings from this in vitro study to those found clinically provided evidence for the clinical relevance of the clot lysis model [56].

In the HisproUK clot lysis study, increasing the mini dose of tPA in the combination had no effect on the lysis time, a finding consistent with tPA's fibrinolytic function being limited to the initiation of lysis. The doses of tPA and HisproUK in the synergistic combination caused no fibrinogenolysis, in contrast to that which was caused by the doses need of either activator used alone to induce a similar lytic effect [53].

\section{Conclusions}

Intravascular thrombosis remains a leading cause of death and disability for which thrombolysis is the only pharmacological remedy. The thrombolytic, tPA, has become essentially synonymous with thrombolysis but its use, or that of one of its longer half-life derivates, has been declining due to its inadequate efficacy in AMI, incompatibility with PCI, limited efficacy and risk of ICH in ischemic stroke, and too high a bleeding risk for most 
patients with venous thromboembolism. Instead, intra-arterial devices have become the treatment of choice in AMI are becoming more frequently used in ischemic stroke as well. The resort to these time-consuming methods to treat very time-sensitive conditions is a reflection on the inadequacy of current thrombolysis.

In retrospect, the conclusion that the results of the tPA mega trials "should be taken as evidence that any mortality differences... between different fibrinolytic regimens are unlikely to be large" [16] was a misinterpretation. Instead, the evidence suggests that it is the fibrin-specific mode of action of tPA which restricted its plasminogen activation thereby giving a non-specific activator like SK, with no such restrictions, an efficacy that was essentially comparable but for different reasons. Therefore, the impression of comparability reflected the fibrinolytic limitations of tPA monotherapy.

The biological plasmin-mediated pathway is the basis of all therapeutic thrombolysis and in nature it utilizes both plasminogen activators, tPA and proUK, in a sequential combination rather than only one. Using this paradigm, a synergistic thrombolytic effect was obtained from fractional doses of the activators both in vitro as well as in a clinical study of AMI.

The other activator, proUK, was unstable in plasma at pharmacological concentrations and a more stable single site substitution mutant was designed to replace it. Preclinical testing showed that HisproUK was comparably synergistic as proUK with a much reduced liability for bleeding complications due to a unique property that its enzymatic form, HisUK, was inhibited by C1INH in plasma.

The combination of mini-dose tPA followed by an infusion of a moderate dose of HisproUK is expected to provide more effective therapeutic thrombolysis with a significantly lower risk of ICH, capable of administration without any delay in stroke. Other indications include facilitated PCI, and the treatment of venous thromboembolism.

\section{Acknowledgments}

Conflict of interest The author is the Scientific Officer of TSI, a company that is developing HisproUK.

Open Access This article is distributed under the terms of the Creative Commons Attribution 4.0 International License (http://creativecommons.org/licenses/by/4.0/), which permits unrestricted use, distribution, and reproduction in any medium, provided you give appropriate credit to the original author(s) and the source, provide a link to the Creative Commons license, and indicate if changes were made.

\section{References}

1. ISTH Steering Committee for World Thrombosis Day (2014) Thrombosis: a major contributor to global disease burden. Thromb Hemost 12:843-852
2. Virchow R (1856) Thrombose und Embolie. Gefassentzundung und septische infektion. Gesammelte Abhandlungen zur Wissenschaftlichen Medicin (in German), vol 219. Von Meidinger \& Sohn, Frankfurt am Main, p 732

3. Afilalo J, Roy AM, Eisenberg MJ (2009) Systematic review of fibrinolytic-facilitated percutaneous coronary intervention: potential benefits and future challenges. Can J Cardiol 25:141-148

4. Kleindorfer D, Lindsell CJ, Brass I, Koroschetz W, Broderick JP (2008) National US estimates of recombinant tissue plasminogen activator use: ICD-9 codes substantially underestimates. Stroke 39:924-928

5. Konstantinides SV (2014) Fibrinolysis for patients with intermediate-risk pulmonary embolism. N Engl J Med 370:1402-1411

6. Campbell BC, Mitchell PJ, Kleinig TJ, Dewey HM, Churilov L, Yassi $N$ et al (2015) Endovascular therapy for ischemic stroke with perfusion-imaging selection. N Engl J Med 372:1009-1018

7. Berkhemer OA, Fransen PSS, Beumer D, van den Berg LA, Lingsma HF, Yoo AJ et al (2015) A randomized trial of intraarterial treatment for acute ischemic stroke. N Engl J Med 372:11-20

8. Goyal M, Demchuk AM, Menon BK, Eesa M, Rempel JL, Thornton $\mathrm{J}$ et al (2015) Randomized assessment of rapid endovascular treatment of ischemic stroke. N Engl J Med 372:1019-1030

9. Schrijver AM, van Leersum Fioole B, Reijnen MM, Hoksbergen AW, Vahl AC et al (2015) Dutch randomized trial comparing standard catheter-directed thrombolysis and ultrasound-accelerated thrombolysis for arterial thromboembolic infrainguinal disease. J Endovasc Ther 22(1):87-95

10. Rijken DC, Collen D (1981) Purification and characterization of the plasminogen activator secreted by human melanoma cells in culture. J Biol Chem 256:7035-7041

11. Voskuilen M, Vermond A, Veeneman GH, van Boom JH, Klasen EA, Zegers ND et al (1987) Fibrinogen lysine residue A $\alpha 157$ plays a crucial role in the fibrin-induced acceleration of plasminogen activation, catalyzed by tissue-type plasminogen activator. J Biol Chem 262:5944-5946

12. Husain SS, Lipinski B, Gurewich V (1981) Rapid purification of high affinity plasminogen activator from human plasma by specific adsorption on fibrin-celite. Proc Natl Acad Sci (USA) 78:4265-4269

13. Pennica D, Holmes WE, Kohr WJ, Harkins RN, Vehar GA, Ward CA et al (1983) Cloning and expression of human tissue-type plasminogen activator cDNA in E. coli. Nature 301:214-221

14. Chesebro JH, Knatterud G, Roberts R Borer J, Cohen LS, Dalen J et al (1987) Thrombolysis in myocardial infarction (TIMI) trial, Phase I: a comparison between intravenous tissue plasminogen activator and intravenous streptokinase. Circulation 76:142-154

15. Gruppo Italiano Per Lo Studio Della Sopravvivenze Nell'Infarto Miocardico (1990) GISSI-2 A factorial randomised trial of alteplase versus streptokinase and heparin versus no heparin among 12,490 patients with acute myocardial infarction. Lancet 336:65-71

16. ISIS-3 Third International Study of Infarct Survival Collaborative Group (1992) ISIS-3: a randomised comparison of streptokinase $v s$ tissue plasminogen activator $v s$ anistreplase and of aspirin plus heparin $v s$ aspirin alone among 41,299 cases of suspected acute myocardial infarction. The Lancet 339:65-71

17. The GUSTO Investigators (1993) An international randomized trial comparing four thrombolytic strategies for acute myocardial infarction. N Engl J Med 329:673-682

18. Ridker PM, Marder VJ, Hennekens CH (1993) Large-scale trials of thrombolytic therapy for acute myocardial infarction: GISSI-2, ISIS-3, and GUSTO-1. Ann Intern Med 119:530-536

19. Brophy JM, Joseph L (1995) Placing trials in context using Bayesian analysis. GUSTO revisited by Reverend Bayes. J Am Med Assoc 273:871-875 
20. Pannell R, Black J, Gurewich V (1988) The complementary modes of action of tissue plasminogen activator (t-PA) and prourokinase (pro-UK) by which their synergistic effect on clot lysis may be explained. J Clin Invest 81:853-859

21. Marder VJ, Shulman NR, Carroll WR (1969) High molecular weight derivatives of human fibrinogen produced by plasmin. I. Physicochemical and immunological characterization. J Biol Chem 244:2111-2119

22. Wiman B, Wallen $P$ (1977) The specific interaction between plasminogen and fibrin. A physiological role of the lysine binding site in plasminogen. Thromb Res 10:213-222

23. Komrowica E, Kolev K, Machovich R (1998) Fibrinolysis with des-kringle derivatives of plasmin and its modulation by plasma protease inhibitors. Biochemistry 37:9112-9118

24. Yakovlev S, Makogonenko E, Kurochkina N, Nieuwenhuizen W, Ingham K, Medved L (2000) Conversion of fibrinogen to fibrin: mechanism of exposure of tPA- and plasminogen-binding sites. Biochemistry 39:15730-15741

25. Hoylaerts M, Rijken DC, Lijnen HR, Collen D (1982) Kinetics of the activation of plasminogen by human tissue plasminogen activator. Role of fibrin. J Biol Chem 257:2912-2919

26. Harpel PC, Chang TS, Verderber E (1985) Tissue plasminogen activator and urokinase mediate the binding of Glu-plasminogen to plasma fibrin I. Evidence for new binding sites in plasmindegraded fibrin I. J Biol Chem 260:443 (abstr)

27. Varadi A, Patthy L (1984) Beta (Leu121-Lys122) segment of fibrinogen is in a region essential for plasminogen bonding by fibrin fragment E. Biochemistry 23:2108-2112 (abstr)

28. Liu J, Gurewich V (1992) Fragment E-2 from fibrin substantially enhances pro-urokinase-induced glu-plasminogen activation. A kinetic study using a plasmin-resistant mutant pro-urokinase (A158-rpro-UK). Biochemistry 31:6311-6317

29. Fleury V, Lijnen HR, Angles-Cano E (1993) Mechanism of the enhanced intrinsic activity of single-chain urokinase-type plasminogen activator during ongoing fibrinolysis. J Biol Chem 268:18554-18559

30. Petersen LC (1997) Kinetics of reciprocal pro-urokinase/plasminogen activation. Stimulation by a template formed by the urokinase receptor bound to poly (D-lysine). Eur J Biochem 245:316-323

31. Liu J, Pannell R, Gurewich V (1992) A transitional state of prourokinase which has a higher catalytic efficiency against gluplasminogen than urokinase. J Biol Chem 267:15289-15292

32. Gurewich V, Pannell R, Broeze RJ, Jao JI (1988) Characterization of the intrinsic fibrinolytic properties of pro-urokinase through a study of plasmin resistant mutant forms produced by site specific mutagenesis of lysine 158. J Clin Invest 82: 1956-1962

33. Rijken DC, Hoylaerts M, Collen D (1982) Fibrinolytic properties of one-chain and two-chain human extrinsic (tissue-type) plasminogen activator. J Biol Chem 257:2920-2925

34. Pannell R, Gurewich V (1992) A comparison of the rates of clot lysis in a plasma milieu induced by tissue plasminogen activator (t-PA) and rec-pro-urokinase: evidence that t-PA has a more restricted mode of action. Fibrinolysis $6: 1-5$

35. Suenson E, Lützen O, Thorsen S (1984) Initial plasmin-degradation of fibrin as the basis of a positive feed-back mechanism in fibrinolysis. Eur J Biochem 140:513-522

36. Liu J, Gurewich V (1991) A comparative study of the promotion of tissue plasminogen activator and pro-urokinase-induced plasminogen activation by fragments D and E-2 of fibrin. J Clin Invest 88:2012-2017

37. Marder VJ, Sherry S (1988) Thrombolytic therapy: current status. N Engl J Med 318:1512-1520
38. Montoney M, Gardell SJ, Marder VJ (1995) Comparison of the bleeding potential of vampire bat salivary plasminogen activator versus tissue plasminogen activator in an experimental rabbit model. Circulation 91:1540-1544

39. Gurewich V, Pannell R, Simmons-Byrd A et al (2006) Thrombolysis versus bleeding from hemostatic sites by a prourokinase mutant compared with tissue plasminogen activator. J Thromb Hemost 4:1559-1565

40. PRIMI Trial Study Group (1989) Randomized double-blind trial of recombinant pro-urokinase against streptokinase in acute myocardial infarction. Lancet 1:863-867

41. Gurewich V, Pannell R (1987) Synergism of tissue-type plasminogen activator (t-PA) and single-chain urokinase-type plasminogen activator (scu-PA) on clot lysis in vitro and a mechanism for this effect. Thromb Hemost 57:372-378

42. Collen D, De Cock F, Demarsin E Lijnen HR, Stump DC (1986) Absence of synergism between tissue-type plasminogen activator (tPA), single-chain urokinase-type plasminogen activator (scuPA) and urokinase on clot lysis in a plasma milieu in vitro. Thromb Hemost 56:35-39

43. Zarich SW, Kowalchuk GJ, Weaver WD, Loscalzo J, Sassower M, Manzo K, Byrnes C, Muller JE, Gurewich V for the PATENT Study Group (1995) Sequential combination of thrombolytic therapy for acute myocardial infarction: results of the prourokinase and t-PA enhancement of thrombolysis (PATENT) trial. J Am Coll Cardiol 26:374-379

44. Rapaport E (1993) GUSTO: assessment of the preliminary results. J Myocard Ischemia 5:15-24

45. Husain SS, Gurewich V, Lipinski B (1981) Purification of a new high MW single chain form of urokinase (UK) from urine. Thromb Hemost 46:1-478 (abstr)

46. Pannell R, Gurewich V (1986) Pro-urokinase: a study of its stability in plasma and a mechanism for its selective fibrinolytic effect. Blood 67:1215-1223

47. Michels R, Hoffmann H, Windeler J, Barth H, Hopkins G (1995) A double-blind multicenter comparison of the efficacy and safety of saruplase and urokinase in the treatment of acute myocardial infarction: report of the SUTAMI Study Group. J Thromb Thrombolysis $2: 117-124$

48. Tebbe U, Michels R, Adgey J, Boland J, Caspi A, Charbonnier B et al (1998) Randomised, double-blind study comparing saruplase with streptokinase therapy in acute myocardial infarction: the COMPASS equivalence trial. J Am Coll Cardiol 31: 487-493

49. Weaver WD, Hartmann JR, Anderson Reddy PS, Sobolski JC, Sasahara AA (1994) New recombinant glycosylated prourokinase for treatment of patients with acute myocardial infarction. J Am Coll Cardiol 24:1242-1248

50. Pannell R, Gurewich V (1987) The activation of plasminogen by single-chain urokinase or by two-chain urokinase-a demonstration that single chain urokinase has a low catalytic activity (pro-urokinase). Blood 69:22-26

51. Sun Z, Jiang Y, Liu BF, Tang W, Chen Y, Gurewich V et al (1997) Identification of a flexible loop (297-313) of urokinasetype plasminogen activator, which helps determine its catalytic activity. J Biol Chem 272:23818-23823

52. Liu JN, Tang W, Kung W, Pannell R, Sarmientos P, Gurewich V et al (1996) A site-directed mutagenesis of pro-urokinase which substantially reduces its intrinsic activity. Biochemistry 35: 14070-14076

53. Liu JN, Liu B, Sun Z, Zhang J, Chen Y, Gurewich V et al (2002) A prourokinase mutant which induces highly effective clot lysis without interfering with hemostasis. Circ Res 90: $757-763$ 
54. Pannell R, Kung W, Gurewich V (2007) C1-inhibitor prevents non-specific plasminogen activation by a prourokinase mutant without impeding fibrin-specific fibrinolysis. J Thromb Hemost 5:1047-1054

55. Gurewich V, Pannell R (2009) Recombinant human C1-inhibitor prevents non-specific proteolysis by mutant prouPA during optimal fibrinolysis. Thromb Hemost 102:279-286
56. Pannell R, Li S, Gurewich V (2015) Highly effective fibrinolysis by a sequential synergistic combination of mini-dose tPA plus low-dose mutant proUK. Plos One 10(3):e122018. doi:10.1371/ journal.pone.0122018 